\title{
TRES ARGUMENTOS KEPLERIANOS CONTRA EL UNIVERSO HOMOGÉNEO
}

\author{
Francisco Javier Luna L.*
}

Este texto pretende analizar tres argumentos esgrimidos por Johannes Kepler en su polémica contra la cosmología de Giordano Bruno. Se ha considerado que existe una diferencia fundamental entre Bruno, quien hizo de la idea de un universo infinito la base de su filosofía natural, y Kepler, quien optó por un universo finito y concéntrico para su sistema astronómico. A pesar de que existen diferencias entre los dos autores mencionados, yo sostengo que también se dio un progresivo acercamiento de Kepler a algunas tesis de Bruno.

Palabras clave: cosmología, infinito, astronomía, ciencia moderna, física celeste.

\section{THREE KEPLERIAN ARGUMENTS AGAINST THE HOMOGENEOUS UNIVERSE}

\begin{abstract}
This paper aims to analyze three arguments wielded by Johannes Kepler in his polemic against the cosmology of Giordano Bruno. It has been found that there is a fundamental difference between the approach of Bruno, who made the idea of an infinite universe based on his natural philosophy, and Kepler, who opted for a concentric finite universe for his astronomical system. Although there are differences between the two authors mentioned, I argue that there is also a progressive approach of Kepler to some Bruno's theses.
\end{abstract}

Keywords: cosmology, infinity, astronomy, modern science, celestial physics.

* Universidad Nacional Autónoma de México (UNAM), Ciudad de México, México. Correo electrónico: lechuza@unam.mx 
Hace más de medio siglo aleXandre koyré estableció una incompatibilidad entre la metafísica de Giordano Bruno (1548-1600), concretamente el tema del universo infinito, y la astronomía heliocéntrica de Johannes Kepler (1571-1630). Aunque ambos autores partían de principios copernicanos, el académico francés afirmó que Kepler nunca habría podido aceptar la infinitud del cosmos, pues en "el trasfondo metafísico último de su pensamiento [...] Kepler sigue siendo un aristotélico". Si bien la argumentación básica de Koyré es firme, habría que establecer ciertos matices en su tajante separación, pues, aunque es cierto que el astrónomo sajón de Weil der Stadt nunca aceptó explícitamente las tesis más radicales de Bruno y también que sus investigaciones astronómicas se iniciaron con una base aristotélica-tolemaica, propia de su época, ello no implica que sus ideas no conocieran diversas etapas, o que él mismo no fuera uno de los principales destructores de la propia cosmología aristotélica.

Retornando a ese problema, la finalidad de este escrito es hacer un análisis de tres argumentos que Kepler esgrimió en contra de las tesis de Bruno como parte de la polémica sobre la naturaleza, orden y geometría del universo, y revisar sus presupuestos e implicaciones. Cada argumento corresponde a una etapa distinta del pensamiento de Kepler, lo que demuestra que las ideas de Bruno estaban lejos de ser letra muerta y seguían acechando como una posibilidad epistémica válida para fundamentar una cosmología. Aun sin Bruno, es posible interpretar los argumentos del Sajón como un debate que incluso terminó por modificar el enfoque de Kepler, como acontece en un diálogo vivo.

Antes de avanzar en ese análisis convendría reseñar brevemente la concepción cosmológica inicial de Kepler, así como el grado de conocimiento que tuvo de la obra de Bruno. El primer esbozo del sistema cosmológico de Kepler apareció en su temprano libro titulado: Prodromus Dissertationum Cosmographicum continens Mysterium cosmographicum (Precursor de consideraciones cosmográficas conteniendo el misterio del universo, 1596), el cual es una curiosa mezcla de cosmología tradicional, especulación geométrica y copernicanismo. La tesis central del Prodromus es que el Sol es el centro del

1 Koyré, Alexandre, Del mundo cerrado al universo infinito, trad. de Carlos Solís, Siglo XXI, Madrid, 1999, pp. 88-89. 
cosmos y motor del movimiento universal, tesis sostenida por el hecho de que la velocidad orbital de los planetas, desde la perspectiva copernicana, es inversamente proporcional a su distancia al astro, así cada planeta es progresivamente más lento hasta llegar a la inmovilidad aparente de las Fijas. Complemento de la idea anterior es que la proporción de las distancias entre planetas se explica, según el joven suabo, por la existencia de un patrón puramente geométrico que delimita los lugares ${ }^{2}$ y que sigue las pautas de los sólidos perfectos, llamados platónicos o pitagóricos:

Es mi intención, lector, demostrar que [...] el Creador Óptimo Máximo, al crear el mundo móvil y en la disposición de los cielos, se atuvo a los cinco cuerpos regulares que han sido tan famosos desde los días de Pitágoras y Platón hasta los nuestros, y también que en función de su naturaleza ajustó su número, sus proporciones y la razón de sus movimientos ${ }^{3}$.

Aunque las líneas anteriores las escribió Kepler apenas con veinticuatro años, el resto de su obra se guió por esas dos ideas: (1) la existencia de una centralidad del Sol, concebido como fuente motriz cósmica y (2) la existencia de un patrón geométrico perfecto en las proporciones entre los planetas, el que explica sus distancias y también su número ${ }^{4}$. Por implicación, el cosmos kepleriano era finito, y esto fue dado por demostrado en el Prodromus ${ }^{5}$. Esa primera obra sostenía la idea de que sólo las figuras regulares son parte de la geometría del cosmos, y por lo tanto no sólo el infinito físico sino hasta los conjuntos matemáticos infinitos son tenidos igualmente por irracionales ${ }^{6}$. Tan importantes eran esas dos ideas básicas que el propio Kepler sigue ateniéndose a ellas aún después de haber realizado el grueso de sus principales descubrimientos astronómicos. Así, en la segunda edición del Prodromus (1621), el suabo escribió:

He aqui que durante estos 25 años me ha resultado provechoso este principio del que ya entonces estaba firmemente convencido, a saber, que las matemáticas son la causa de las cosas naturales (doctrina combatida por Aristóteles en múltiples lugares) porque Dios creador tuvo consigo desde la eternidad a la matemática como arquetipo en una abstracción simplísima y divina, incluso de las propias cantidades, considerada materialmente ${ }^{7}$.

2 Los Loci, en sentido aristotélico. Cfr. ArIsó Cruz, Albert, "La noción de lugar en la metafísica aristotélica", Thémata, Revista de Filosofía, 45, 2012, pp. 35-50.

3 Kepler, J., El secreto del Universo, trad. de Eloy Rada, Alianza, Madrid, 1992, p. 65. [Prodromus, "Praefatio ad lectorem", §6, 1-6].

4 En época de Kepler únicamente se conocían 6 planetas: Mercurio, Venus, Tierra, Marte, Júpiter y Saturno.

5 Kepler de hecho da por demostrado en su primera obra que "Mundum igitur totum figura claudi sphaerica, abunde satis disputavit Aristoteles (libro II de coelo)". KePler, J., Astronomi Opera Omnia, vol. I, Christian Frisch, Heyder \& Zimmer, Frankfurt, 1863, p. 123. [Prodromus, II, \$20, 29-31].

6 "Pero desterremos las líneas rectas y las superficies en tanto que infinitas y por ende absolutamente incapaces del orden de este mundo finito, armonioso y bellísimo." KePLer, J., El secreto del Universo, op. cit., p. 95. [Prodromus, II, §21,16-18]. Kepler niega que los conjuntos infinitos matemáticos sean parte de la arquitectura del cosmos. 
En el Prodromus nada delata un conocimiento propio de las tesis de Bruno, y a lo sumo podemos ver una cierta simpatía hacia las especulaciones matemáticas de Nicolás de Cusa y una referencia a la famosa cita hermética de Dios como esfera infinita ${ }^{8}$. El nombre de Bruno comienza a aparecer en las obras y la correspondencia de Kepler a partir de 1606. En una carta de 1608, el suabo se lamentaba del suplicio y la ejecución del Nolano, ahí leemos: "Bruno fue quemado en Roma, según dijo Wackher, tras soportar con firmeza los suplicios. Por afirmar la vanidad de todas las religiones, la identidad de Dios con el mundo, y del punto con el círculo" . Como el mismo Kepler lo cita, su fuente principal sobre Bruno fue Matthaeus Wackher de Wackhenfels, quien además poseía algunas obras del Nolano que seguramente le sirvieron de consulta. Miguel Ángel Granada sostiene que Kepler ya formaba parte del círculo de Wackher desde antes de $1604^{10}$; aunque algunos rastros en la Astronomia nova, como el progresivo cambio de enfoque cosmológico, podrían sugerir que esa cercanía se inicia hacia 1602.

\section{La caverna en medio de un mar de estrellas}

La primera obra en que Kepler discute las tesis cosmológicas de Bruno es De Stella Nova in Pede Serpentari (De la estrella nueva en el pie del Serpentario, 1606), aparecida para explicar la nova de 1604-1605. En ese libro, Kepler, ya investido como Astrónomo Imperial, primero reconoció que el modelo heliocéntrico anula el argumento de Aristóteles contra la infinitud y también aceptó que la infinitud del cosmos se puede deducir de los atributos divinos:

Aristóteles había demostrado por el movimiento la finitud del Mundo. Copérnico, genuinamente, privó de movimiento a la esfera de las Fijas, permitiendo que sea infinita [infinitam esse patiatur]. Como se sabe, esta idea fue defendida por el desdichado Jordano Bruno. Y también, de forma nada oscura, de modo dubitativo, por William Gilbert en su libro De magnete por lo demás admirable. El sentimiento religioso de Gilbert era tan fuerte que, según él, si comprendes con recto discernimiento el infinito poder de Dios,

7 Kepler, J., El secreto del Universo, op. cit., pp. 125 y 126. [Prodromus, II, §21,16-18], pp. 125 y 126. [Prodromus, XI, nota b]. La cita anterior también nos revela que la distancia con Aristóteles fue, ciertamente, creciendo.

8 "Dei trinuni imago in sphaerica superficie, Patris scilicet in centro, Filii in superficie, Spiritus in aequalitate $\sigma \chi \varepsilon \sigma \varepsilon \omega \varsigma$ inter puncturo et ambitum. Nam quae Cusanns circulo, alii forte globo tribuerent, ea epo soli sphaericae superficiei arrogo". KePLeR, J., Astronomi Opera Omnia, vol. I, p. 122. [Prodromus, II, §19, 20-24].

9 "Brunum Romae crematum ex Dominino Wackherio didici, ait constanter suplicum tulisse. Religionum omnium uanitatem asseruit, Deum in mundum in circulos in puncta conuertit". Carta de Kepler a Bregger de 1608 citada por Hansch, Michael Gottlieb, Epistolae ad Joannem Kepplerum mathematicum caesareum scriptae, Vol. 2., Frankfurt \& Leipzig, 1718, p. 266. La última parte de la cita nos muestra que Kepler relacionaba a Bruno con Cusa y la geometría divina. El conocimiento de Bruno que poseía Kepler dista de ser superficial; en opinión de Domenico Berti, Kepler estudió y llegó a apreciar las obras de Bruno. Véase: BerTi, Domenico, Vita di Giordano Bruno di Nola, Paravia, Turín, 1868, p. 8.

10 Matthaeus Wackher von Wackenfels fue diplomático de Rodolfo II, un converso al catolicismo y seguidor de Bruno. Granada afirma que Wackher poseía copias del Spaccio, Del infinito y de los tres diálogos latinos, que pudo haber prestado a Kepler. Granada, Miguel Ángel, "Kepler and Bruno on the infinity of the universe and solar system", Journal for the History of the Astronomy, vol. XXXIX, 2008, p. 472. 
entonces se demuestra que ha hecho infinita a la materia del mundo ${ }^{11}$.

El problema de la infinitud queda como una posibilidad abierta en el heliocentrismo, pues al atribuirle el movimiento diario de las Fijas a la rotación de la Tierra y no a la última esfera, ésta puede ser infinitamente grande; más aún, al haber demostrado Tycho Brahe que no podían existir las esferas sólidas ${ }^{12}$, la esencia de la tesis del Estagirita queda derrumbada. Además existía el problema de la ausencia de paralaje, pues si se admitía el punto de vista copernicano, esto implicaba la existencia de distancias interestelares inmensas. Si bien el problema del paralaje no implica la existencia de un universo infinito, sí la de uno extraordinariamente grande. Toda lo anterior podía ser tomada como evidencia en favor de Bruno. Sin embargo, Kepler no podía aceptar la infinitud, pues contravenía la unicidad del sistema planetario (tesis 2) y el corolario de un universo homogéneo negaba la centralidad solar (tesis1) $)^{13}$. Es por ello que Kepler escribió:

Bruno ve al mundo como infinito, tanto a las estrellas Fijas como a los mundos, y hace de nuestra región móvil uno de los innumerables mundos [...] Su simple pensamiento trae no sé qué horror oculto, uno se encuentra a sí mismo vagando en la inmensidad, cuyos límites, cuyo medio [centro], así como todo lugar, son negados ${ }^{14}$.

Al reconocer que el heliocentrismo negaba los fundamentos de la tesis finitista, Kepler se encontraba en la difícil posición de argumentar contra Bruno, quien derivó su cosmología desde las mismas bases copernicanas que tenía Kepler, respetando esas bases -incluso reforzándolas de ser posible- pero negando las implicaciones del Nolano. El camino del suabo en De Stella fue el mismo trazado por Copérnico, primero mostrar que la distancia entre la Tierra y las Fijas ${ }^{15}$ es proporcionalmente menor que la velocidad de rotación de la última esfera según el modelo tolemaico ${ }^{16}$. Una vez hecho este prolegómeno, el suabo pasa a atacar la homogeneidad de un universo infinito utilizando una visión del cosmos derivada de su idea del Sol como motor del Mundo, ya presentada en el Prodromus:

11 "Quod ARISTOTELES ex motu finitum mundum demonstraverit: COPERNICUS verò fìxarum Sphaeram, adempto motu, infinitam esse patiatur. Itaque defendit illam infelix ille JORDANUS BRUNUS: nec obscurè asseruit, speciedubitantis, et GUGLIELMUS GILBERTUS, libro de Magnete, caetera praeclarissimo, eligiosum tamen affectum eo demonstravit, quòd existimaret non alia re rectius intelligi infinitam Dei potentiam, quàm si infinitum mole conderet mundum". KePLeR, J., Gesammelte Werke, vol. I, Ed. Max Caspar, C. H. Beck'sche Verlangbuchhandlung, Munich, 1938, p. 253. [De Stella Nova, XXI, §105, 42-45].

12 Heidarzadeh, Tofigh, A History of Physical Theories of Comets, From Aristotle to Whipple, Springer, Dordrecht, 2008, pp. 42-43.

13 "Porque dicho universo es infinito y, por tanto, sin centro y sin límite." BRuno, Giordano, Sobre el universo infinito y los mundos, trad. de Ángel Cappelletti, Terramar, La Plata, 2008, p. 9. [De l'infinito, III]. Bruno después amplía estas ideas en el poema latino De inmenso.

14 "Sed Brunus ita infinitum facit mundum ut quod sunt Stellae fixae, tot mundos, et hanc nostram regionem mobilium unum ex innumerabilibus mundis faciat [...] Quae sola cogitatio, nescio quid horroris occulti prae se fert; dum errare sese quis deprehendit in hoc immenso; cujus termini, cuius medium, ideoque et certa loca, negatura". KePler, J., Gesammelte Werke, vol. I, p. 253. [De Stella Nova, XXI, §106, 8-17].

15 Kepler calculó una distancia mínima a las estrellas con un paralaje tomado del margen de error de las observaciones a simple vista (2") dándole como resultado $2,160.000$ radios terrestres $(2,786.4$ millones de kilómetros). 
La perfección del Mundo es el movimiento, que para él es casi una especie de vida. Se requieren tres cosas para que exista el movimiento: el Motor, el Móvil y el Lugar. El Motor es el Sol; los Móviles son todo lo comprendido desde Mercurio hasta Saturno; el Lugar es la última esfera de las estrellas Fijas ${ }^{17}$.

Adoptando una perspectiva claramente aristotélica, para el suabo la perfección del cosmos es tener movimiento, el cual necesita de un motor (el Sol), un móvil (los Planetas) y un espacio (el marco de las Fijas). Aunque en estricto sentido ya no existe una "esfera", Kepler conserva un sentido del concepto para hablar de un borde último del universo físico y, a pesar de que dicho borde se encuentra a una distancia enorme, si es mensurable, también es finito. Un presupuesto de la cosmología kepleriana de ese momento era que las estrellas no comparten la misma naturaleza que el Sol, sino que se les asigna el papel de límite de lo finito. Las estrellas no eran soles para el suabo, pues eso llevaría a la existencia de múltiples motores y a abrir la posibilidad a múltiples sistemas planetarios. Así, en ese momento, para Kepler existen tres tipos de objetos astronómicos: el Sol, los planetas y las estrellas, cada uno con su propia naturaleza de acuerdo a su lugar cósmico.

Para medir ese borde estelar, Kepler usó un argumento especulativo en función de su imagen motriz ${ }^{18}$ y calculó el tamaño del universo en $34,077,066^{2} / 3$ radios terrestres ${ }^{19}$. La pasmosa cantidad no acerca al astrónomo alemán al universo infinito del Nolano ${ }^{20}$, al contrario, Kepler insistía que una medida, por enorme que sea, es lo opuesto a lo ilimitado. Esto tampoco puede demostrar la finitud, pero sí alcanza para argumentar contra la homogeneidad; lo que le preocupa al suabo es la falta de un centro (cuius medium) y, por ende, de lugares (loci). Según su visión de la astronomía, Kepler afirmaba que existía cierta cavidad entre las estrellas, alejada de todas las demás, con un único sol en medio:

Admitamos por principio que las Fijas se expanden hacia fuera infinitamente, pero esto sería tan cierto como que existe una cavidad en lo profundo, marcadamente distinta del espacio que existe entre las estrellas Fijas y el Sol; entonces, si por casualidad alguien viera esta cavidad única, ignorando que existen sus ocho corpúsculos orbitando una pequeñisima área central, cómo son, cuántos son y de qué clase son, sin embargo, por

16 La hipotética velocidad de la esfera de las Fijas según el modelo ptolemaico de 2,625 radios por hora (3.386 millones de $\mathrm{Km} / \mathrm{h}$ ).

17 "Mundi perfectio est motus, quae ejus quasi quaedam vita est. Ad motum tria requiruntur, Motor, Mobile, Locus. Motor est Sol: Mobilia sunt à Mercurio usque ad Saturnum: Locus est extima fixarum Sphaera". KePLer, J., Gesammelte Werke, vol. I, p. 234. [De Stella Nova, XVI, §85, 27-30].

18 El diámetro de los objetos móviles (la distancia entre el Sol y Saturno) es el medio proporcional de diámetro del Sol y el diámetro de las estrellas Fijas [De Stella Nova, XVI]. El argumento es especulativo porque presupone un modelo heliocéntrico y una proporción entre las distancias planetarias y astrales.

19 Brahe, en su Progymnasmata, afirma que la distancia es de 14,000 radios; por ende, De Stella es una polémica a varios frentes, como afirma Westman. Véase: Westman, Robert S., The Copernican Question: Prognostication, Skepticism, and Celestial Order, University of California Press, Berkeley, 2011, pp. 394 y 398.

20 "Realmente no hay proporción entre la gala suprema de Dios con la exigüidad de nuestro mundo." KePLER, J., Gesammelte Werke, vol. I, p. 237. [De Stella Nova, XVI, §88, 15-16]. 
ese espacio vacio, comparado con la región esférica circundante, llenísima de estrellas, deberá concluir sin dudas, que de una manera u otra ahi es un lugar especial, ciertamente la principal cavidad del Mundo ${ }^{21}$.

Este primer argumento consiste en demostrar la existencia de una región no homogénea y se funda sobre los datos sensoriales directos para trazar un mapa estelar. Kepler especula que las estrellas podrían ser todas del mismo tamaño real y, por tanto, el tamaño aparente de las estrellas está en proporción con su distancia ${ }^{22}$. En base a eso, el suabo calcula la distribución de las estrellas y demuestra que un hipotético astrónomo extraterrestre en Orión vería un cielo muy diferente al de nuestra noche. Según la distribución calculada, la distancia del Sol a las estrellas más próximas es mayor que la existente entre las estrellas, de modo que su hipotético astrónomo:

vería al principio un ininterrumpido inmenso mar de estrellas, entrelazadas a la vista; a partir de ese lugar, mientras más se eleva la mirada, paulatinamente las estrellas se dispersan más y más, mirando hacia el vórtice se verían las mismas [estrellas] que nosotros vemos, pero la mitad de grandes y el doble de próximas que nosotros las vemos aquí...23.

De forma evidente en el relato anterior, la imagen del cielo en Orión no es la misma que nosotros vemos. El primer argumento gira por completo en torno al problema de la homogeneidad, allí el objetivo del suabo era demostrar que la región donde habitamos difiere de las demás y constituye una especie de caverna. En cambio, el problema del infinito no es abordado directamente, Kepler no polemiza contra éste ni entra al terreno teológico ${ }^{24}$, sino que se queda en el plano sensorial; su conclusión es que: "es por lo tanto cierto, que desde el Sol a los Planetas el Mundo es finito y de cierto modo ahuecado. Lo que resta, la metafísica lo completará" ${ }^{25}$. El punto de escisión estaba en admitir sólo los datos sensoriales ${ }^{26}$,

21 "Nam, esto inter principia, extendantur fixxae in infinitum foras; verum tamen est, in hoc intimo sinu, cavitatem esse insignem, distinctamque ab intervallis, quae sunt inter fixas stellas, immani proportionis dissimilitudine: sic, ut si cui hanc solam cavitatem contingeret inspicere, ignoranti etiam de octo corpusculis, circa hujus spacij centrum, angustissimo quidem intervallo, circumvolantibus, quae, qualia, quotve sint: tamen is ex sola hac vacuitate, comparatâ cum circumambeunte sphaerica regione, stellis refertissimâ, omnino esset conclusurus, peculiarem aliquem locum hîc esse; adeoque praecipuum mundi sinum”. Ibidem, p. 253. [De Stella Nova, XXI, $\$ 106,32-40]$.

22 Ibidem, pp. 254-256. [De Stella Nova, XXI, §107 y 108].

23 "Videret ille primò perpetuum quoddam quastrenasi mare ingentium stellarum, sese mutuò contingentium ad visum: inde, quo magis oculos levaret sursum, hoc minores videret stellas, hocque minus sese contingentes; sed jam paulatim magis atque magis sparsas; circa ipsum verò suum verticem, easdem nobiscum videret; sed duplo minores, tantoque invicem propiores, quàm nos illas videmus". Ibidem, p. 254. [De Stella Nova, XXI, §107, 1015]

24 Kepler comprende que la tesis de la omnipotencia divina conlleva abrir las puertas a considerar el cosmos infinito, o al menos indeterminado, así que evita entrar directamente en ese debate. Sin embargo, la otra vía, la lógica, que se desprende del argumento de la flecha de Lucrecio que repite Bruno en: Bruno, G., Sobre el universo infinito y los mundos, p. 9. [De l'infinito, "Argomento del primo dialogo"]. Kepler prefirió omitir esta posibilidad, incluso en el Dissertatio, donde ya habla de otros autores como Demócrito, e insiste en la tesis de Gilbert. 
por lo cual no podemos plantear ni siquiera la cuestión de la infinitud, que queda relegada de forma vaga al ámbito metafísico.

\section{La paradoja de la noche oscura}

El segundo argumento contra Bruno lo elabora Kepler en la Dissertatio cum Nuncio Sidereo (Conversación con el mensajero sideral, 1610), donde discute las novedades que Galileo ha observado con su cannocchiale. Precisamente una de esas novedades es que los halos brillantes de las estrellas son una mera ilusión óptica ${ }^{27}$, y por lo que los supuestos del argumento del De Stella son refutados. Kepler, lejos de desanimarse, afirma que los descubrimientos del cannocchiale confirman sus ideas finitistas, pues creía que ellos podían demostrar su creencia, anteriormente presupuesta, en la diferencia de naturalezas entre las estrellas y el Sol.

Es interesante resaltar que, en un principio, el suabo temía que Galileo hubiera descubierto nuevas tierras girando alrededor de las estrellas, lo cual habría cuestionado su geometría del $\operatorname{cosmos}^{28}$, como también la diferencia que él sostenía entre la singularidad del Sol y las Fijas. Grande fue su alivio cuando pudo leer que los nuevos astros eran lunas y no tierras, como confiesa a Galileo:

todo me encuentro contentísimo de haber nacido en gran medida de nuevo gracias a tus trabajos. Si hubieras encontrado planetas girando en torno a una de las innumerables fijas, ya tenía yo reservadas cadenas y cárcel junto a las innumerabilidades de Bruno, o incluso más bien el exilio en aquel infinito ${ }^{29}$.

Pero al confirmar que son nuevas lunas, Kepler afirmó no haber encontrado ningún problema con su sistema físico o su hipótesis geométrica, incluso especuló que las nuevas lunas muestran que otros planetas están habitados, admitiendo la uniformidad cósmica de Bruno, pero sólo en nuestro sínodo planetario ${ }^{30}$. En su escrito, el suabo aceptó de buena

25 "Est igitur certum, introrsum versus Solem et Planetas finitum esse mundum, et quodammodo excavatum. Quod reliquum est, Metaphysica superstruat". KePler, J., Gesammelte Werke, Vol. I, Ed. Max Caspar, C. H. Beck'sche Verlangbuchhandlung, Munich, 1938, p. 256. [De Stella Nova, cap. XXI, §109, 15-16].

26 Bruno ya había explicado que: "No hay sentido que vea al infinito, no hay sentido de quien se pueda exigir esta conclusión, porque el infinito no puede ser objeto de los sentidos, $\mathrm{y}$, en consecuencia, quien pretende conocerlo por medio de los sentidos es semejante a quien quiera ver con sus ojos la sustancia y la esencia, y quien negase por eso la cosa, por cuanto no es sensible o visible, llegaría a negar la propia sustancia y ser." Bruno, G., Sobre el universo infinito y los mundos, op. cit., p. 30. [De l'infinito, I].

27 Kepler acepta de inmediato este hecho: "El anteojo no quita en la Tierra nada que las estrellas tengan en el cielo, sino que elimina de la retina toda la luz que sobra". KePLER, J., Conversación con el mensajero sideral, trad. de Carlos Solís, Alianza, Madrid, 2007, p. 169. Incluso Kepler aporta la teoría óptica necesaria para explicar ese fenómeno en su libro Ad Vitellionem paralipomena, quibus astronomiae pars optica traditus (1604).

28 "Así, me puse a meditar para mí como podría aumentarse en algo el número de planetas dejando a salvo mi Misterio cosmográfico...". KePler, J., Conversación con el mensajero sideral, op. cit., p. 131. Como se puede leer la principal preocupación de Kepler era salvaguardar su tesis juvenil.

29 Ibidem, p. 173 
fe el testimonio galileano, y además explicó correctamente el funcionamiento de la doble lente y confirmó que la eliminación del halo brillante no es una ilusión óptica, sino lo contrario. El cannocchiale comprobó que las estrellas producen luz, mientras que los planetas son cuerpos oscuros que sólo la reflejan, lo que llevó a Kepler a aceptar que las estrellas son luminosas o que, "para decirlo con palabras de Bruno, que aquellos son soles y estos son tierras o lunas" 32 . A pesar de ese reconocimiento, el suabo creyó descubrir una diferencia ontológica entre las Fijas y el Sol, al considerar que ese enorme conjunto de estrellas no podía iluminar la noche con la misma claridad que una sola podía iluminar el día. Así, para Kepler la misma disminución del diámetro angular demostraba que las estrellas tienen una naturaleza diversa al Sol: "sobre la primera afirmación relativa al seno interior del mundo, véase los argumentos extraídos de la multitud de las fijas que como un muro rodean realmente esta caverna, y de la claridad de nuestro Sol superior a la de las

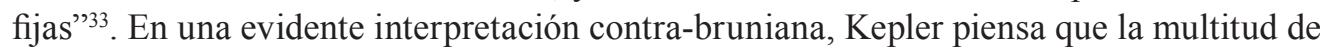
nuevas Fijas muestra cómo éstas conforman una especie de muro exterior que delimita el globo del Mundo y las luminarias sólo eran un sistema de balizas para indicar ese límite:

Pues bien, si tomamos sólo mil de las fijas, no siendo ninguna de ellas mayor de un minuto (si bien en los registros la mayoría son mayores), y si las juntásemos todas en una superficie redonda, igualarian (y aun superarian) el diámetro solar. ¿Cuánto más habría de superar en tamaño visible a la imagen del orbe solar diez mil pequeños discos de estrellas reunidos en uno? Si esto es cierto, y si dichos soles son del mismo tipo que nuestro Sol ¿por qué todos esos soles no superan en resplandor a este Sol nuestro? ${ }^{34}$.

El argumento kepleriano plantea una paradoja conocida hoy como "paradoja de Olbers", aunque bien podría llamarse de $\mathrm{Kepler}^{35}$, y que se resume con la pregunta: ¿por qué la noche es oscura? La existencia de innumerables pequeños discos estelares que bien podían ser reunidos y superar el tamaño aparente del Sol, pero que no iluminaban tan intensamente como éste, era motivo de incomprensión si ambos son de la misma naturaleza. El problema ataca tanto la tesis de infinitud como de la uniformidad del cosmos, pues si

30 Kepler propone una causa magnética, tomada de Gilbert, para explicar el movimiento de las Lunas. Aunque esto modifica un poco la idea del Sol como único motor celeste, no modifica radicalmente la imagen del cosmos.

31 "Se podría decir, por supuesto, que la Tierra tiene una luna porque está habitada. Así, si los planetas tienen lunas deberían estar también habitados. ¿Por qué no habrían de estarlo? Según Kepler, que acepta las enseñanzas de Cusa y Bruno para nuestro mundo, no hay razones para negar esta posibilidad." KoYré, A., Del mundo cerrado al universo infinito, op. cit., p. 78. Como dice Kepler "se torna evidente que estos nuevos planetas no se orientan primariamente a los que nos hallamos en la Tierra, sino que sin duda se relacionan con las criaturas joviales que viven en el globo de Júpiter". KePLeR, J., Conversación con el mensajero sideral, op. cit., p. 180.

32 Kepler, J., Conversación con el mensajero sideral, op. cit., p. 170. Cfr. Bruno, G., Sobre el universo infinito y los mundos, op. cit., pp. 107-108. [De l'infinito, cap. III].

33 KePler, J., Conversación con el mensajero sideral, op. cit., p. 183.

34 Ibidem, p. 171.

35 Aunque Thomas Digges había adelantado el problema cuando consideró la última esfera infinita y con infinitas "luces", para él realmente no había una paradoja, pues pensaba que la inmensa distancia hacía que no viéramos más que unas pocas. Digges, Thomas, A Perfit Description of the Celestiall Orbs, citado por HARRISON, Edward, Darkness at Night: A Riddle of the Universe, Harvard University Press, Cambridge, p. 37. 
existen infinitas estrellas distribuidas en capas uniformes y éstas han estado brillando durante un tiempo infinito, no deberían existir lugares oscuros.

La distancia a las estrellas no resuelve la paradoja, pues a mayor distancia mayores dimensiones tienen los cuerpos reales de las estrellas. Tampoco se puede apelar, según Kepler, a un oscurecimiento por el medio, a un éter que absorbiera la luz pues: "las vemos [las estrellas] con sus centelleos y con sus diversas formas y colores" ${ }^{\prime 36}$. Es evidente que esto último no es una idea completa, pero no le correspondía a Kepler demostrar la inexistencia de algo para probar su tesis. La paradoja de la noche ha demostrado ser un argumento implacable a lo largo del tiempo, pues sigue jugando un papel en las cosmologías contemporáneas que presuponen la igualdad de naturaleza entre el Sol y las estrellas ${ }^{37}$. Para Kepler la paradoja mostraba que, precisamente, dicha igualdad no existía y que el universo contiene un lugar especial en su centro con una estrella también especial.

Kepler termina su argumento contra el Nolano afirmando que si existieran múltiples sistemas solares, éstos sólo podrían ser meras copias de la perfección del sistema geométrico, en cuyo caso son reiteraciones innecesarias, o bien una multitud de sistemas que "diferirán en el orden, tipo y perfección de las figuras"38, en cuyo caso demostrarían imperfecciones indignas de Dios.

Antes de continuar, aclaremos un poco cómo cambió el panorama astronómico para ese momento. El inicio de la astronomía con telescopio confirmó que importantes tesis geocentristas como la perfección de los cielos estaban equivocadas, también mostró que los astros rotan y que los planetas son de naturaleza tenebrosa, y que los planetas, al menos los inferiores, orbitan al Sol. Pero para decepción de los copernicanos no logró demostrar que la Tierra es móvil al medir un paralaje. Esto sólo podía significar que la Tierra estaba fija o bien que las estrellas estaban todavía más lejos y que eran más grandes de lo imaginado. La idea de que esos gigantescos soles sólo sirvieran para delimitar la cavidad móvil interior resultó difícil de digerir.

36 KePLeR, J., Conversación con el mensajero sideral, op. cit., p. 171. La solución moderna del polvo estelar resuena de un modo peculiarmente similar, postular objetos intermedios que absorban la luz no resuelve el problema, pues infinitas estrellas, brillando por tiempo infinito, hacen que todos los cuerpos opacos terminen por emitir ondas electromagnéticas. HARRISOn, Edward, Darkness at Night: A Riddle of the Universe, op. cit., pp. $110 \mathrm{y}$ ss.

37 Las diversas soluciones de la paradoja generalmente pasan por negar la infinitud de la materia o la eternidad del tiempo, lo cual parece refutar la premisa de un universo infinito. Las soluciones históricas que han respetado las premisas empezaron por Edmund Halley, quien planteó la posibilidad de que la luz se podría debilitar a un ritmo mayor que el cuadrado de la distancia, hasta propuestas actuales basadas en una distribución fractal del cosmos. Al respecto, puede consultarse: JAKI, Stanley L., "Olbers', Halley’s, or Whose Paradox?", Americal Journal of Physics, 35, 3, pp. 200-210.

38 Kepler, J., Conversación con el mensajero sideral, op. cit., p. 184. 


\section{El problema del apilado de naranjas}

Después de sopesar esas consideraciones, el propio Kepler tuvo que admitir que las estrellas compartían prácticamente todas las propiedades del Sol. Quizás esto fue lo que lo animó a no ahondar en su paradoja y buscar un nuevo argumento que refutara la homogeneidad y salvara la unicidad del sistema solar. El tercer argumento contra Bruno y la homogeneidad lo formula Kepler en su obra de plena madurez Epitome Astronomiae Copernicae (Compendio de astronomía copernicana, 1618-1621). En el libro primero del Epitome, Kepler aborda de nueva cuenta las principales tesis cosmológicas de Bruno "y otros antiguos" ${ }^{39}$ sobre la infinitud del cielo. Pero a diferencia de lo presentado en la Dissertatio, en el Epitome por fin se acepta que el Sol sea una más de las innumerables estrellas y "si nos parece más grande y más brillante, es porque está más cerca que las Fijas" ${ }^{\prime 40}$. El resultado de ello sería que el sistema solar en nada se diferenciaría del resto del universo, excepto en que, de nueva cuenta, Kepler insistía en que estaba ubicado justo en el centro absoluto del cosmos.

El argumento en sí puede parecer sumamente extraño la primera vez que se escucha: Kepler afirmó que si el universo fuera homogéneo, deberíamos poder ver a doce estrellas "enormes" $"$ en comparación con el resto. Estas estrellas deberían estar colocadas en un patrón en el cielo y tener distancias proporcionales unas de otras. Como evidentemente esto no ocurre, Kepler concluyó que el universo no es homogéneo. El argumento regresa a la vieja asunción de que las estrellas más cercanas son de mayor magnitud aparente ${ }^{42}$. Pero detrás del planteamiento existe una muy interesante cuestión cosmológica y atómica. Si uno se pregunta por qué precisamente doce estrellas, la respuesta es: para formar los ángulos de un icosaedro. Lo que Kepler estaba pensando era que los sistemas planetarios eran como burbujas o globos que se agrupaban unos junto a otros, y al estar en el espacio debían de agruparse de una manera determinada por el axioma de la simplicidad y eficiencia de la naturaleza. Y la manera más eficiente posible de empacar esferas es apilándolas en un patrón de icosaedros ${ }^{43}$.

39 "Et veterum aliqui". Kepler, J., Astronomi Opera Omnia, vol. VI, p. 136. [Epitome, I: "Principiorum doctrinae sphaericae", Pars secunda: de figura coeli].

40 "Nec Sol hic noster aliud erit, quam una ex fixis, nobis major et clarior visa, quia propior quam fixae". Idem.

41 "Tunc apparerent nobis paucae aliquae fixae ingentes" Ibidem, p. 137. [Epitome, I: "Principiorum doctrinae sphaericae", Pars secunda: de figura coeli."].

42 "However, he omits the part which considered the possibility that stars of the same magnitude might be at different distances and gives no reference to his earlier and more detailed account". FieLD, J.V., Kepler's geometrical cosmology, The Anthlone Press, Londres, 1988, p. 25.

43 Kepler es el primero, que sabemos, en ocuparse matemáticamente del problema y conjeturó (sin llegar a una demostración completa) que el empaquetado de icosaedros es el más eficiente. La conjetura de Kepler, a veces llamada "el problema del apilado de naranjas", quedó como un problema abierto hasta 1998, que fue demostrado por fuerza bruta mediante ordenador. Véase: Hales, Thomas C., "A proof of the Kepler conjecture", Annals of Mathematics, Vol. 162, 3, 2005, pp. 1065-1185. 
En la cuestión de los apilados se esconde un problema fundamental del atomismo. Bruno ya había señalado que los átomos no tienen partes, pero de alguna forma deben conectarse unos con otros, por ello introdujo el concepto de termini. Ahora, al ser circulares, Bruno ensayó las distribuciones de contacto entre los átomos (De triplici, II, xiii). El Nolano propuso distribuciones en cuadrado y en triángulo, para ilustrar cómo se forman las mallas de átomos. Pero olvidó un punto fundamental, señalado por Kepler en el Prodromus: que las formas materiales están en tres dimensiones; pues el círculo "no existe si no es en el plano, esto es, salvo que una superficie esférica o un globo sean cortados por un plano, no existirá ningún círculo" ${ }^{\$ 4}$. Así, el problema de empacado de Kepler es, en realidad, una cuestión de unión entre esferas, que esconde un modelo de ordenamiento macro y microcósmico.

En el Strena Seu de Nive Sexangula (Regalo navideño o sobre la nieve de seis ángulos), Kepler fue categórico sobre el problema de empacado:

En todo el Universo los globos iguales se ordenan entre sí de dos modos posibles en su receptáculo, así como también tienen dos modos de ordenarse en cualquier otro plano. Porque si reúnes globos errantes en un mismo plano horizontal y los encierras en un lugar más estrecho, de modo que se toquen unos a otros, o tienden a ordenarse de forma triangular o bien cuadrangular. ${ }^{45}$

Dichas distribuciones son las mismas señaladas por Bruno, en la forma cuadrangular cada esfera está rodeada de otras cuatro, en la triangular, en cambio, está rodeada por seis. En caso de compresión, una formaría un cuadrado y otra un hexágono. Kepler señaló que no existe el quincuángulo pues "no puede retener la igualdad"46 (continuar el patrón), mientras que el sexángulo es una forma duplicada del triángulo. Con esas bases, Kepler fue mucho más allá de lo planteado por Bruno, pues transformó el problema a tres dimensiones. El sajón razonaba que si las Fijas fueran soles con sistemas planetarios propios, serían una especie de globos, y si éstos tuvieran una distribución isotrópica y homogénea, entonces deberían estar empaquetados eficientemente. Esta explicación, Kepler no la expresa en el Epitome, pero se presupone de todo su argumento.

Este último argumento seguía, en apariencia, las líneas del primero, ya que se asumía que la información sensorial estaba directamente relacionada con la distribución geométrica real de los cuerpos celestes. Sin embargo, el argumento del Epitome tiene cierta peculiaridad, como agudamente señaló Field: "Kepler a menudo refiere a su lector hacia sus otros trabajos, pero su silencio en este caso podría indicar que él no estaba ya satisfecho con lo que había escrito en el De Stella Nova"47. Esta insatisfacción podemos relacionarla

44 KePler, J., El secreto del Universo, op. cit., p. 93. [Prodromus, cap. II].

45 KePler, J., Regalo festivo o De la nieve sexángula, trad. de Vidal González, Libros en Casa, Málaga, 2010, p. 91. 46 Idem.

47 "Kepler often does refer his reader to his other works so his silence in this instance may indicate that he was no 
con el cambio en su consideración de la naturaleza estelar, presupuesta como diferente en el De Stella, argumentada en la Dissertatio y finalmente aceptada en el Epitome. Si a lo anterior le sumamos que el problema del empacado trata a las estrellas como globos, podemos reconstruir la idea de que Kepler intenta, con este último argumento, salvar una última diferencia entre nuestra estrella y el resto de las Fijas: el hecho de que la nuestra posee un espacio asociado, es decir, su propio sínodo planetario que junto con la estrella crean una caverna, como dice en el De Stella, o un globo. Si existieran en una distribución homogénea esos globos, uno en cada estrella, entonces se comprende mejor la exigencia del empaquetado cósmico. Aunque Kepler se mantuvo sin aceptar la posibilidad de un universo isotrópico y homogéneo, el grado de rechazo hacia la idea de un universo infinito se fue mitigando, desde el rechazo total de su primera obra, conforme avanzó su polémica con Bruno, hasta la aceptación de las tesis de Bruno para nuestro sistema planetario ${ }^{48}$ y también para la naturaleza estelar. Y aunque Kepler no admitió explícitamente un universo infinito, sí admitió sin reservas que el infinito matemático es una pieza esencial de la geometría del cosmos. Como el propio Kepler escribió en su segunda edición del Prodromus:

Desterremos las líneas rectas y las superficies en tanto que infinitas y por ende absolutamente incapaces del orden [ordinis minime capaces] de este mundo finito y armonioso. iQué error! ¿Las apartaremos del Mundo [las infinitas lineas rectas y curvas]?, y además por su propio fuero las readmití en el Harmonices. ¿Por qué, pues, las ibamos a arrojar? ¿Quizás porque son infinitas y así excesivamente susceptibles de orden? Pero no eran tanto ellas mismas, cuanto mi ignorancia de entonces, que compartía con la mayor parte, lo que me hacía incapaz de atribuirles algún orden. Así, en el libro I del Harmonices no sólo expuse un cierto principio de elección entre estos infinitos, sino que también puse de manifiesto el más hermoso orden entre ellos. Pues ¿por qué habríamos de eliminar las lineas del arquetipo del universo, siendo así que Dios las incluyó en la obra misma, esto es, los movimientos de los planetas? ${ }^{49}$ *

longer satisfied with what he had written in De Stella Nova". FIELD, J.V., Kepler's geometrical cosmology, op. cit., p. 25.

48 En realidad no sólo admitió la uniformidad de los fenómenos entre planetas, sino que admitió que ciertos principios, como la generación y corrupción, se aplican universalmente. Como explica Boner: "Central to Kepler's celestial physics was an effort to reconfigure the cosmos [...] abandoning the hierarchical view of heavens and the passive Earth, Kepler applied a single causal system to flatten, so to speak, the two spheres". Boner, Patrick, "Kepler's vitalistic wiew of the heavens" en Granada, Miguel Ángel, Novas y cometas entre 1572 y 1618, Publicacions i edicions de la Universitat de Barcelona, Barcelona, 2012, p. 184. Aunque la visión vitalista de Kepler no necesariamente proviene de Bruno, si es una afinidad entre ambos que Koyré omite con su imagen del suabo como un astrónomo apegado sólo a los datos observacionales y aquello que se pueda derivar lógicamente de ellos.

49 KePler, J., El secreto del Universo, op. cit., pp. 101 y 102. [Prodromus, cap. II, nota a]. Aunque Kepler utiliza las técnicas de cuadraturas de Arquímedes en sus posteriores obras, incluso el nombre del matemático griego está ausente del Prodromus. En esta primera obra utiliza prácticamente sólo los Elementos de Euclides, mientras que para el final de su carrera es uno de los pioneros del cálculo.

* Artículo recibido: 20 de noviembre de 2014. Aceptado: 17 de diciembre de 2014. 


\section{Bibliografía}

Arisó Cruz, Albert, "La noción de lugar en la metafísica aristotélica", Thémata Revista de Filosofía, No. 45, 2012, pp. 35-50.

Berti, Domenico, Vita di Giordanno Bruno di Nola, Paravia e comp., Turín, 1868.

Boner, Patrick, “Kepler's vitalistic wiew of the heavens" en: Granada, Miguel Ángel, Novas y cometas entre 1572 y 1618, Publicacions i edicions de la Universitat de Barcelona, Barcelona, 2012, pp. 165-193.

Bruno, Giordano, Sobre el universo infinito y los mundos, trad. de Ángel Cappelletti, Terramar, La Plata, 2008.

FIELD, J.V., Kepler's geometrical cosmology, The Anthlone Press, Londres, 1988.

Granada, Miguel Ángel, "Kepler and Bruno on the infinity of the universe and solar system", Journal for the History of the Astronomy, vol. XXXIX, 2008, pp. 469-495.

Hales, Thomas C., "A proof of the Kepler conjecture", Annals of Mathematics, Vol. 162, 3, 2005, pp. 1065-1185.

Hansch, Michael Gottlieb, Epistolae ad Joannem Kepplerum mathematicum caesareum scriptae, vol., Frankfurt \& Leipzig, 1718.

Harrison, Edward, Darkness at Night: A Riddle of the Universe, Harvard University Press, Cambridge, 1987.

Heidarzadeh, Tofigh, A History of Physical Theories of Comets, From Aristotle to Whipple, Springer, Dordrecht, 2008.

Jaki, Stanley L., “Olbers', Halley's, or Whose Paradox?”, Americal Journal of Physics, 35, 3, pp. 200-210.

Kepler, Johannes, Astronomi Opera Omnia, vol., Christian Frisch, Heyder \& Zimmer, Frankfurt, 1863.

Conversación con el mensajero sideral, trad. de Carlos Solís, Alianza, Madrid, 2007. 
Francisco JAVIER Luna L.

-, El secreto del Universo, trad. de Eloy Rada, Alianza, Madrid, 1992.

Gesammelte Werke, Vol. I, Ed. Max Caspar, C. H. Beck'sche Verlangbuchandlung, Munich, 1938.

, Regalo festivo o De la nieve sexángula, trad. de Vidal González, Libros en Casa, Málaga, 2010.

Koyré, Alexandre, Del mundo cerrado al universo infinito, trad. de Carlos Solís, Siglo XXI, Madrid, 1999.

Westman, Robert S., The Copernican Question: Prognostication, Skepticism, and Celestial Order, University of California Press, Berkeley, 2011. 